\title{
Hyperandrogenism, Insulin Resistance, Acanthosis Nigricans Syndrome
}

National Cancer Institute

\section{Source}

National Cancer Institute. Hyperandrogenism, Insulin Resistance, Acanthosis Nigricans

Syndrome. NCI Thesaurus. Code C131008.

A condition characterized by hyperandrogenism, insulin resistance, and acanthosis nig ricans, typically associated with obesity in teenage girls. It is considered to be a subtype of polycystic ovarian syndrome, but may occur in male individuals. Etiology is unclear, but some cases may be associated with mutations affecting the tyrosine kinase domain of the insulin receptor. 